\title{
MINIMALLY INVASIVE PHOTOPOLYMERIZATION IN INTERVERTEBRAL DISC TISSUE CAVITIES
}

\author{
Andreas M. Schmocker*a,b Azadeh Khoushabi ${ }^{\mathrm{b}, \mathrm{c}}$, Benjamin Gantenbein-Ritter ${ }^{\mathrm{d}}$, Samantha Chan ${ }^{\mathrm{d}}$, \\ Harald Marcel Bonél ${ }^{\mathrm{e}}$, Pierre-Etienne Bourban ${ }^{\mathrm{c}}$, Jan Anders Månson ${ }^{\mathrm{c}}$, Constantin Schizas ${ }^{\mathrm{f}}$, \\ Dominique Pioletti ${ }^{\mathrm{b}}$, Christophe Moser $^{\mathrm{a}}$
}

\author{
a Laboratory of Applied Photonics Devices, Microengineering Department, Swiss Federal Institute of \\ Technology Lausanne (EPFL), Station 17, 1015 Lausanne, Switzerland; \\ ${ }^{\mathrm{b}}$ Laboratory of Biomechanical Orthopedics, Institute of Bioengineering, EPFL, Station 19, 1015 \\ Lausanne, Switzerland; \\ ${ }^{\mathrm{c}}$ Laboratory of Polymer and Composite Technology, Institute of Materials, EPFL, Station 12, 1015 \\ Lausanne, Switzerland; \\ ${ }^{\mathrm{d}}$ Tissue \& Organ Mechanobiology Group, Institute for Surgical Technology and Biomechanics, \\ University of Bern, Stauffacherstrasse 78, 3014 Bern, Switzerland; \\ Institute for Diagnostic, Pediatric and Interventional Radiology, Inselspital, University of Bern, \\ Bern, Freiburgstrasse, 3010 Bern, Switzerland \\ ${ }^{\mathrm{f}}$ Centre Hospitalier Universitaire Vaudois, Orthopedic Department, Av. P. Decker 4, 1011 Lausanne, \\ Switzerland
}

\begin{abstract}
Photopolymerized hydrogels are commonly used for a broad range of biomedical applications. As long as the polymer volume is accessible, gels can easily be hardened using light illumination. However, in clinics, especially for minimally invasive surgery, it becomes highly challenging to control photopolymerization. The ratios between polymerizationvolume and radiating-surface-area are several orders of magnitude higher than for ex-vivo settings. Also tissue scattering occurs and influences the reaction.

We developed a Monte Carlo model for photopolymerization, which takes into account the solid/liquid phase changes, moving solid/liquid-boundaries and refraction on these boundaries as well as tissue scattering in arbitrarily designable tissue cavities. The model provides a tool to tailor both the light probe and the scattering/absorption properties of the photopolymer for applications such as medical implants or tissue replacements. Based on the simulations, we have previously shown that by adding scattering additives to the liquid monomer, the photopolymerized volume was considerably increased.

In this study, we have used bovine intervertebral disc cavities, as a model for spinal degeneration, to study photopolymerization in-vitro. The cavity is created by enzyme digestion. Using a custom designed probe, hydrogels were injected and photopolymerized. Magnetic resonance imaging (MRI) and visual inspection tools were employed to investigate the successful photopolymerization outcomes.

The results provide insights for the development of novel endoscopic light-scattering polymerization probes paving the way for a new generation of implantable hydrogels.
\end{abstract}

Keywords: Polymerized medical implant, light scattering, Monte Carlo simulation, cross-linking, injectable hydrogel, in situ photopolymerization, intervertebral disc regeneration, nucleus pulpous replacement 


\section{INTRODUCTION}

Lower-back pain is experienced by 70 to $85 \%$ of the world's population once in their life ${ }^{1}$. The resulting cost in OECD countries is estimated to be up to $\$ 100$ billion per year. The current surgical options for spinal disorders (e.g. intervertebral disc degeneration, general spine degeneration ${ }^{2}$ ) are not very effective ${ }^{3}$. If conservative treatments fail, the gold standard remains spinal fusion after discectomy ${ }^{4,5}$. Although temporarily the pain is alleviated in the short term, problems are often shifted to adjacent vertebral segments in the long run. ${ }^{6}$ Therefore, more physiological solutions are highly warranted, i.e. minimally invasive while re-establishing pre-operation disc-height and joint motion. A considerable amount of effort is directed at replacing the jelly core (the Nucleus Pulpous) of the intervertebral disc (IVD) by a synthetic mechanical implant. A promising replacement option are photopolymerizable hydrogels ${ }^{7}$ because their mechanical properties depend on their cross-linking density, polymer composition, degree of swelling ${ }^{8}$ and polymerization conditions $s^{9,10}$. By understanding and controlling polymerization patterns, local material properties can be engineered (elastic modulus, swelling ratio e.g.), to match the set of requirements for the implant. However, to be implantable, it is essential to better understand minimally-invasive photopolymerization where the ratio between the polymerization-volume and a probe's radiating-surface-area is several orders of magnitude higher than in a usual setting (e.g. a lamp illuminating a surface). Figure 1 illustrates the two settings.
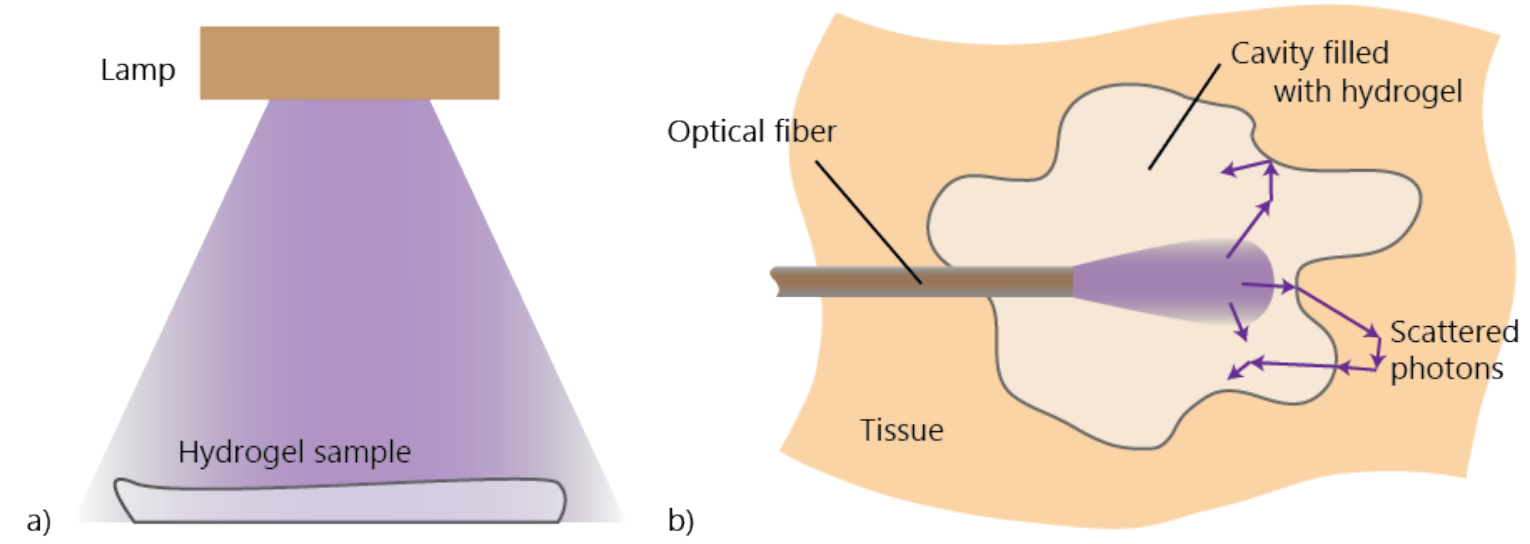

Figure 1 Two types of settings for photopolymerization: a) illumination by lamp, b) implanted hydrogel sample in a tissue cavity illuminated by an optical light guide.

In biotechnology, hydrogels are typically first photopolymerized ex-vivo and then implanted. They are used for a broad range of applications such as drug delivery ${ }^{11}$, 3D tissue scaffolds ${ }^{12}$ and cell-encapsulation ${ }^{13,14}$. In contrast, in situ photopolymerization for in vivo application hasn't been extensively studied.

In this article we report on the modeling of a photopolymerizable hydrogels and their experimental application as a replacement of the interior of the IVD. In the first part a Monte Carlo model for photopolymerization was applied to hydrogels in tissue cavities. In the second part the outcomes of the simulations were experimentally evaluated: cavities were prepared, tailored hydrogels injected and photopolymerization done via a small catheter. The results of the in-situ photo-polymerization were evaluated using macroscopic visual inspection and MRI.

\section{PHOTOPOLYMERIZATION SIMULATIONS}

Previously, a Monte Carlo model for photopolymerization was developed by us ${ }^{15,16}$. We showed that the model was able to compute liquid-to-solid-transitions during the photopolymerization. It was studied how hydrogels photopolymerize in test tubes or in tissue cavities. In this article supplementary results are presented.

\subsection{Computing liquid-solid interfaces}

On liquid-solid-interfaces photons can be reflected or refracted which can alter the final outcome of the reaction. As Monte Carlo simulations are based on photons' probabilities to reach a certain position, the result of a simulation is statistical. Therefore, curves have to be fitted onto these results to construct proper interfaces. Different curve-fitting and 
averaging algorithms were evaluated. Median filters prove to be the most efficient tool to flatten the original Monte Carlo results (fig. 1a). A 2D-median filter was applied on a given amount of voxels or pixels. The bigger the area the stronger was the filtering effect. Areas of $5 \times 5$ to $20 x 20$ voxels were tested (fig. $1 \mathrm{~b}-\mathrm{d}$ ). The 10x10-area lead to similar surface structures as observed during experiments and thus was chosen for the simulations.

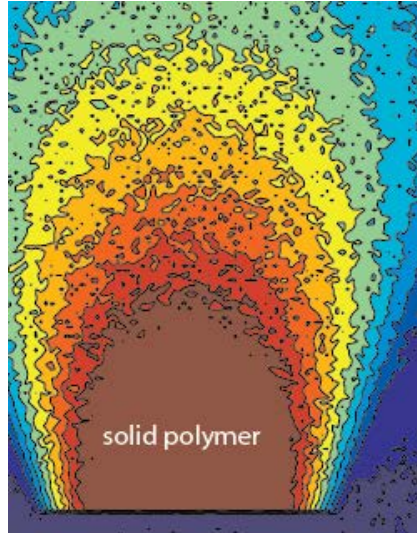

a)

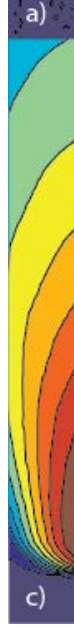

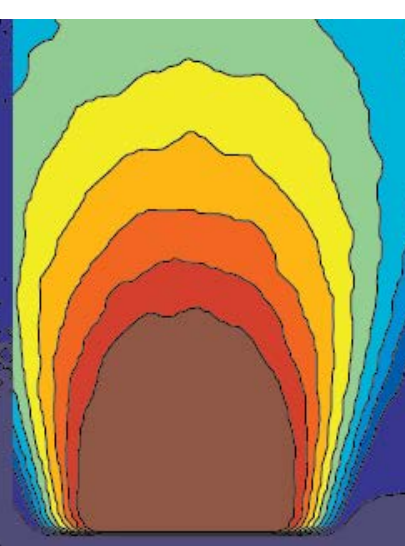

b)

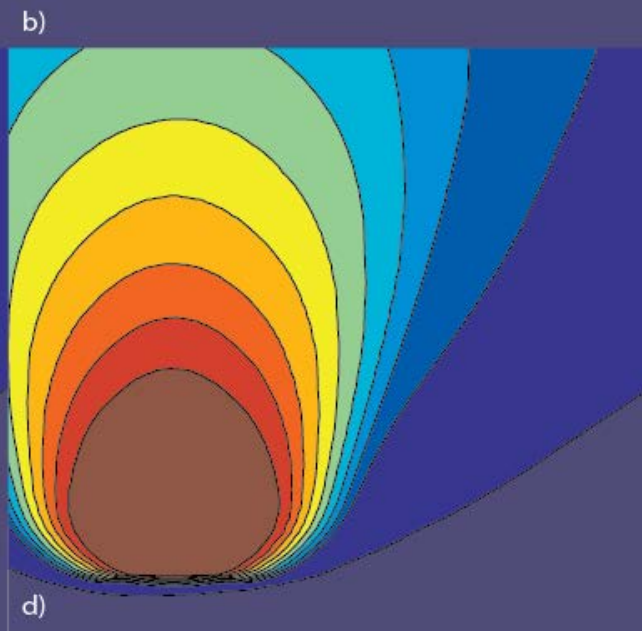

Figure 1 Intensity profiles of an optical fiber illuminating a polymer volume: a) original result of the Monte Carlo simulation b) applying a median filter over $5 \times 5$ voxels, c) 10x10 voxels (used for simulations) and d) 20x20 voxels

Depending on the photopolymerization rate one of the lines is chosen as interface between solid and liquid polymer. This interface is then used to computed refractions or reflections of photons.

\subsection{Changes of scattering and absorption coefficient over time}

By mixing photoinitiator with Intralipid-particles hydrogels with different optical properties were engineered. A transmission setup (fig. 2a) was used to measure these optical properties at $365 \mathrm{~nm}$ wavelength. The hydrogels were injected into liquid chambers where they can be observed throughout the transition from liquid to solid. Water was used as a zero reference. Absorption and scattering coefficients are calculated by separating scattering and absorbing partials and by using Beer's law. Figure 2 illustrates a highly and lowly absorbing hydrogel and the changes of absorption over time: these values were around $\pm 5 \%$ of the mean value and did not show any regular patterns. Similar results were found for the scattering/non-scattering and the absorbing/non-absorbing hydrogels and thus it was concluded that absorption and scattering coefficients did not change significantly over time due to the chemical reaction. 


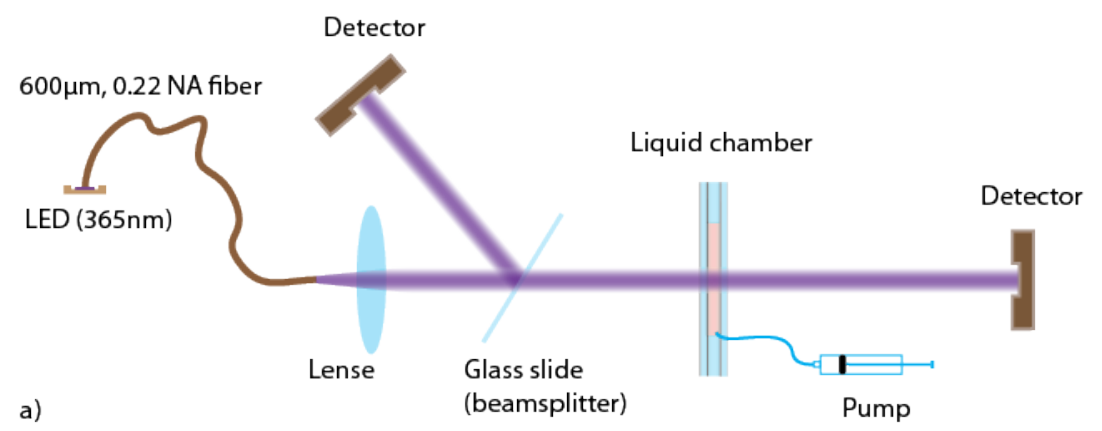

b)

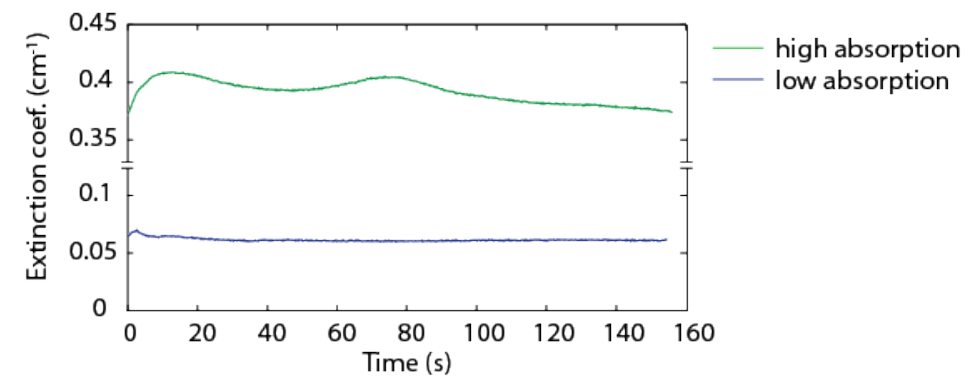

Figure 2 a) transmission setup ${ }^{15}$ used to measure hydrogel samples placed in the liquid chamber, b) extinction coefficient measurement over time of strongly absorbing and lowly absorbing hydrogel.

\subsection{Change of refractive index between liquid and solid}

Whether a photon is reflected or is refracted on the interface between liquid and solid depends on its incident angle and on the relative change of the refractive index $\Delta \mathrm{n}$, which was assumed to be $0.01^{17}$. Using Fresnel's equations each time a photon crossed a liquid-solid-interface its probability to be reflected or refracted was calculated (linear and circular polarizations were averaged). Figure 3 shows the photopolymerized volume over time with and without taking into account reflections/refractions on the liquid-solid interface. No significant change in volume was found.

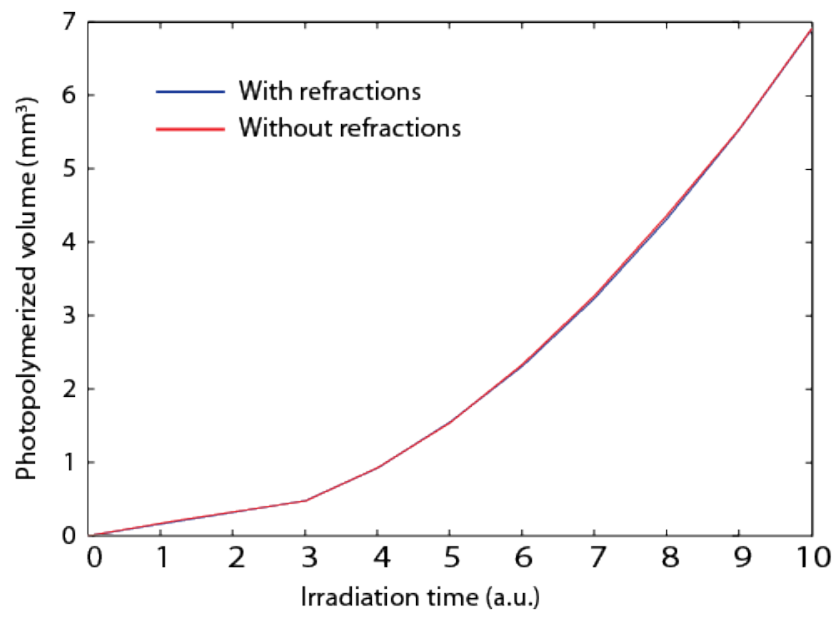

Figure 3 Computed photopolymerized volumes with and without taking into account liquid-solid interfaces between polymerized and unpolymerized hydrogel. The light source was a fiber with $0.22 \mathrm{Na}, 600 \mu \mathrm{m}$ illuminating a volume of initially liquid hydrogel. The photopolymerized (solid) volume was calculated after each time step.

Therefore it was concluded that at a $\Delta \mathrm{n}=0.01$ reflections and refractions can be neglected. 


\subsection{Simulation of photopolymerization in tissue cavities}

Instead of applying the algorithm on liquid-solid-interfaces it can also be applied to simulate polymer-tissue-interfaces. The results of the photopolymerization model is illustrate in figure 4, which shows a tissue cavity in-silico with a hydrogel inside and a light probe placed outside (a) or inside the cavity (b and c). The position of the probe is crucial: Placing the probe a tenth of a mm outside of the cavity results in almost no absorption of light by the hydrogel in the cavity. In b) most of the energy is absorbed by the hydrogel, however, as the 0.22-Na fiber is only able to deliver light within a given cone, absorption takes place only in this cone. A significant portion of light leaves the cavity at the opposite boundary and is again absorbed by the tissue surrounding the hydrogel. By adding Intralipid-particles to the hydrogel (fig. 4c) its scattering coefficient was increased: the lipid-particles reflect the light and lead to a light absorption in the entire cavity: only a small portion of light is lost in the tissue. In c) the scattering coefficient is $10 \mathrm{~cm}^{-1}$, while in a) and $b$ ) it is zero.

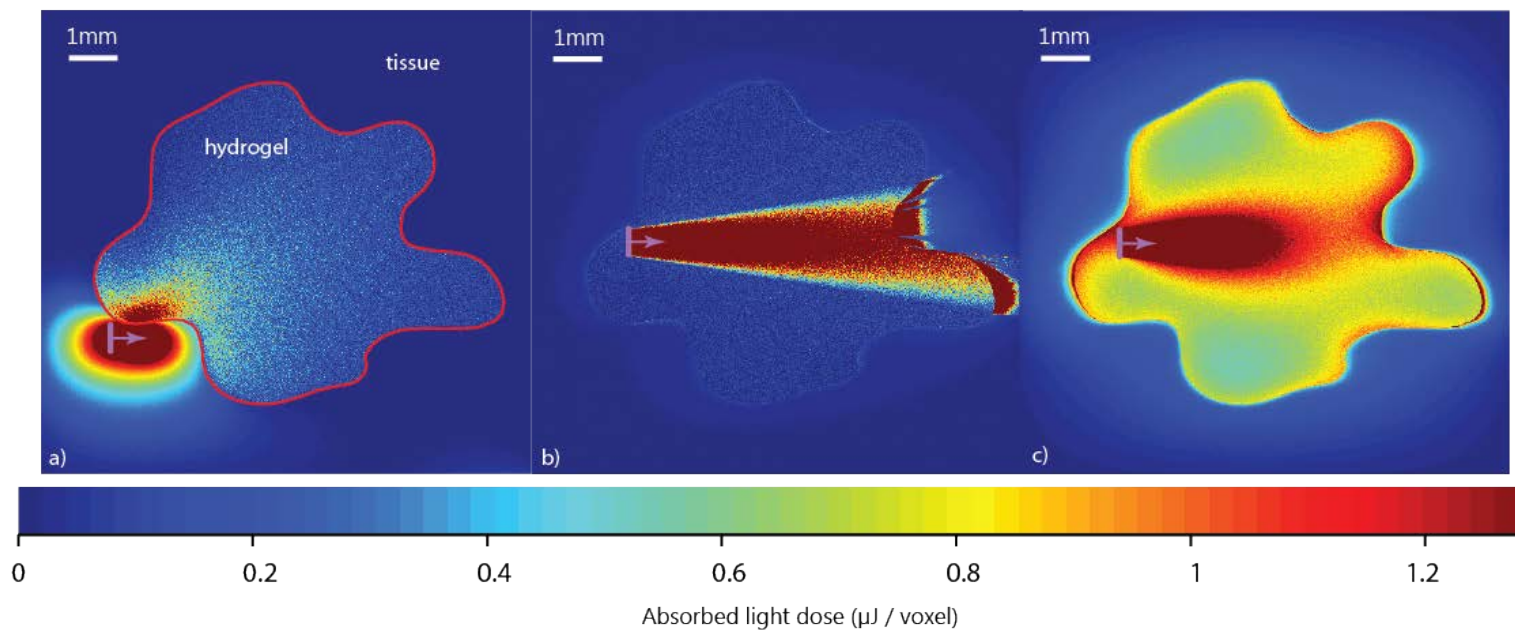

Figure 1 Tissue cavity with initially liquid hydrogel inside illuminated with a $600 \mu \mathrm{m}$ fiber at $6 \mathrm{~mW}$ over ten minutes ${ }^{15}$ : a) the polymerization probe is placed outside the tissue cavity, b) the polymerization probe is inside the cavity, c) probe inside and scattering particles are added to the hydrogel.

The simulations show that for minimally-invasive, in-situ photopolymerization the position of a light probe is essential and that the light absorption of the surrounding tissue is highly relevant. The tissue-absorption can by countered either by illuminating the implanted hydrogel sample in a structured way or by adding scattering particles to the hydrogel itself to achieve a uniform light distribution.

\section{PHOTOPOLYMERIZATION EXPERIMENTS}

\subsection{Light probe, tissue cavities and injections}

A custom fiber-coupled light-source was built by butt-coupling multimode optical fibers ( 0.4 to $1 \mathrm{~mm}$ core) to $365 \mathrm{~nm}$ LED-chips (Nichia Corporation). The resulting fiber provided an output power of 5 to $7 \mathrm{~mW}$. A mercury lamp was used for one test to reach higher power levels at the output of the fiber $(120 \mathrm{~mW})$. The used hydrogel is Poly(Ethylene Glycol) Dimethacrylate $\left(\mathrm{M}_{\mathrm{w}}=6\right.$ and $20 \mathrm{kDa}$, synthesized in-house $\left.{ }^{18}\right)$ mixed with the photoinitiator Irgacure 2959 (BASF) and Intralipids (10 w/v\%, Fresenius Kabi).

Intervertebral discs (IVD) were harvested from fresh bovine tails. To create an artificial cavity within the discs the enzyme Papain is injected ${ }^{19}$. Following the injection the discs are incubated over seven days to activate the Papain (digestion of the Nucleus Pulposus tissue). Several batches of discs are prepared and tested:

1. Papain at 30 and 60 units $/ \mathrm{ml}$, injection and photopolymerization of plain hydrogel, MRI, seven days of cyclic testing, MRI, cell viability tests

2. Papain at 120 units/ml, injection and photopolymerization of plain hydrogel with or without dyes, dissection 
3. Papain at 150 units/ml, injection and photopolymerization of scattering hydrogel, dissection

The amount of injected Papain varies between 100 and around $260 \mu \mathrm{l}$ and hence the size of the created cavity. The volume of injected hydrogel ranges from approximately 150 to $400 \mu \mathrm{l}$.

\subsection{In-situ photopolymerization of intervertebral disc replacements}

For the first batch of IVDs, the illumination time was between $10 \mathrm{~min}$. and 20 min. to ensure complete photopolymerization. Then, photopolymerization was verified using Magnetic Resonance Imaging (MRI). As the MRI images in figure 5 indicate, it was possible to distinguish between a void and a filled cavity.

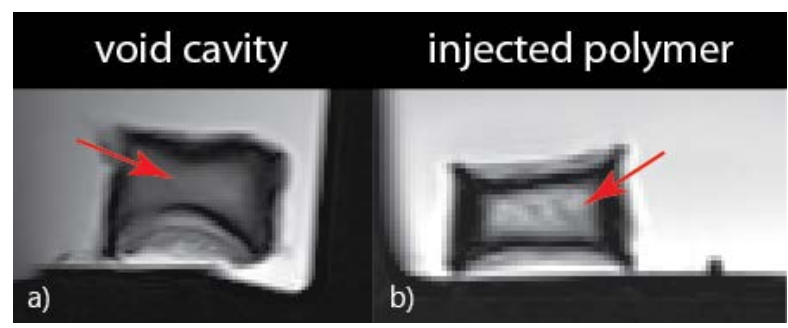

Figure 5 MRI’s T2-signal of the tow samples (batch \#1): a) empty cavity (grey) and b) with injected hydrogel inside (whitish)

However, the MRI could not distinguish between liquid and solid hydrogel. After seven days of cyclic testing (1Hz, per day: $16 \mathrm{~h}$ at an amplitude of $0.6 \mathrm{Mpa}$ and $8 \mathrm{~h}$ at $0.1 \mathrm{Mpa}$ ) of the IVDs no gel could be seen anymore by visual inspection. Hence it remains open whether the injected hydrogels did photopolymerize.

Thus, for the second batch, IVDs were cut open after the injection and photopolymerization of the hydrogel. Due to the transparent nature of the hydrogel (being visually similar to the IVD tissue), hydrogel and tissue were not distinguishable. Therefore, dyes (Methylene blue and Alizarin red) were added to increase the visibility of the hydrogel to the eye. Unfortunately, the dyes diffused over time through the hydrogel to the IVD and prevented a differentiation between hydrogel and tissue. It was also observed that when injecting the liquid hydrogel a small amount was able to leak through the endplates. It was concluded that the hydrogel was not fully polymerized in vitro.

Therefore, Intralipids were added to the third batch to increase the volume of polymerized hydrogel as it is expected from the simulation. To prevent leakage, a thick layer of vertebral bone was left on the bottom and top end of the IVDs. The injection probe was redesigned to increase the precision during injection and illumination. 1 w/v\% of Intralipids were added to the hydrogel to increase the scattering coefficient to $\sim 7 \mathrm{~cm}^{-1}$. After implantation, the hydrogel precursor was illuminated during $60 \mathrm{~min}$ at a power of $6 \mathrm{~mW}$, which resulted in successful and complete photopolymerization of the hydrogel. The turbid Intralipid-hydrogel could easily be distinguished from the IVD tissue. Figure 6 shows an IVD after dissection. The slightly glimmering substance is the implanted hydrogel surrounded by lamellar annulus fibrosus tissue of the IVD.

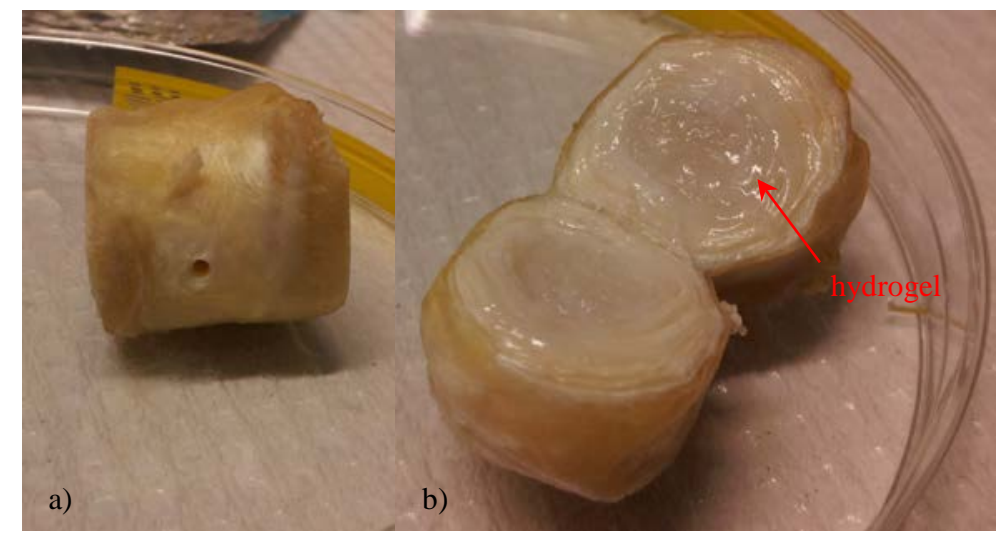

Figure 6 Bovine intervertebral disc after implantation: a) surgeries is performed through a 14 Gauge needle, $400 \mu \mathrm{l}$ of hydrogel were injected and photopolymerized in situ b) transversal cut through the intervertebral disc reveals the solid hydrogel inside the IVD 


\section{DISCUSSION AND CONCLUSION}

A set of factors are decisive for successful in-situ photopolymerization of an implanted scaffold or tissue replacement. 1) Surrounding tissue can absorb a major part of the light illumination. Therefore, the positioning of a light probe is crucial. 2) It is essential to distribute the illumination energy in a controlled way, either by illuminating a material in a structured manner or by adding scatterers to the sample to distribute the light uniformly. 3) An additive is required to judge the success of a photopolymerization procedure. Dyes or X-ray contrast materials diffuse into surrounding tissues and make a differentiation between hydrogel and tissue impossible. The size of Intralipid particles $\left(\sim 100 \mathrm{~nm}^{20}\right)$ seems to match the pores created in the hydrogel during photopolymerization. Thus they remain trapped in the gel and make it visibly distinguishable from tissue.

In this study the illumination duration was kept at 60 min (successful tests) to avoid under-illumination of the hydrogel precursor. For translation into the clinics, this time is not acceptable. Tests have to be conducted to determine the minimal illumination time under the current illumination conditions. It is also possible to increase the illumination intensity or to switch to a biocompatible photoinitiator, which is effective in UVA, violet or blue wavelengths. The structural influence of the Intralipid additive has to be further assessed. The Intralipid may weaken a hydrogel in terms of mechanical stability.

In this article we investigated photopolymerization by means of simulations and experiment. The issues encountered during experimental in-situ polymerization could be explained using the Monte Carlo model for light transport. Beside proper physical access to a tissue cavity, a uniform distribution of light within the cavity seemed to be essential. This issue was resolved by adding Intralipid particles to the hydrogel, making it scattering.

\section{AKNOWLEDGEMENT}

This work was funded by the Swiss National Science Foundation (grant \#10024003165465).

\section{REFERENCES}

[1] Andersson, G.B., “Epidemiological features of chronic low-back pain.,” Lancet 354(9178), 581-5 (1999).

[2] Edeland, H.G., “Some additional suggestions for an intervertebral disc prosthesis.,” Journal of biomedical engineering 7(1), 57-62 (1985).

[3] Schizas, C., Kulik, G., and Kosmopoulos, V., “Disc degeneration: current surgical options.,” European cells \& materials 20, 306-15 (2010).

[4] Hu, R.W., Jaglal, S., Axcell, T., and Anderson, G., “A population-based study of reoperations after back surgery.," Spine 22(19), 2265-2270; discussion 2271 (1997).

[5] Zarghooni, K., Siewe, J., and Eysel, P., “State of the art of lumbar intervertebral disc replacement,” Der Orthopäde 40(2), 141-7 (2011).

[6] Palma, L., Carangelo, B., Muzii, V.F., Mariottini, A., Zalaffi, A., and Capitani, S., "Microsurgery for recurrent lumbar disk herniation at the same level and side: do patients fare worse? Experience with 95 consecutive cases," Surgical Neurology 70(6), 619-621 (2008). 
[7] Eyholzer, C., de Couraça, a B., Duc, F., Bourban, P.E., Tingaut, P., Zimmermann, T., Månson, J. a E., and Oksman, K., "Biocomposite hydrogels with carboxymethylated, nanofibrillated cellulose powder for replacement of the nucleus pulposus.,” Biomacromolecules 12(5), 1419-27 (2011).

[8] Bryant, S.J., Anseth, K.S., Lee, D. a, and Bader, D.L., "Crosslinking density influences the morphology of chondrocytes photoencapsulated in PEG hydrogels during the application of compressive strain.,” Journal of orthopaedic research : official publication of the Orthopaedic Research Society 22(5), 1143-9 (2004).

[9] Anseth, K.S., Bowman, C.N., and Brannon-Peppas, L., "Mechanical properties of hydrogels and their experimental determination.,” Biomaterials 17(17), 1647-57 (1996).

[10] Simms, H.M., Brotherton, C.M., Good, B.T., Davis, R.H., Anseth, K.S., and Bowman, C.N., "In situ fabrication of macroporous polymer networks within microfluidic devices by living radical photopolymerization and leaching.," Lab on a chip 5(2), 151-7 (2005).

[11] An, Y., and Hubbell, J. a, “Intraarterial protein delivery via intimally-adherent bilayer hydrogels,” Journal of Controlled Release 64(1-3), 205-215 (2000).

[12] Bryant, S.J., Nicodemus, G.D., and Villanueva, I., "Designing 3D photopolymer hydrogels to regulate biomechanical cues and tissue growth for cartilage tissue engineering.,” Pharmaceutical research 25(10), 237986 (2008).

[13] Nguyen, K.T., and West, J.L., "Photopolymerizable hydrogels for tissue engineering applications,” Biomaterials 23(22), 4307-4314 (2002).

[14] Burdick, J.A., and Anseth, K.S., "Photoencapsulation of osteoblasts in injectable RGD-modified PEG hydrogels for bone tissue engineering,” Biomaterials 23(22), 4315-4323 (2002).

[15] A. Schmocker, A. Khoushabi, C. Schizas, P.E. Bourban, D.P. Pioletti, C.M., "Photopolymerizable hydrogels for implants: Monte-Carlo modeling and experimental in vitro validation,” Submitted to Journal of Biomedical Optics, (2013).

[16] Schmocker, A., Khoushabi, A., Farahi, S., Pioletti, D., Bourban, P.-E., Manson, J.A., and Moser, C., "Multiscale modeling of photopolymerization for medical hydrogel-implant design,” in SPIE BiOS, A. P. Wax and V. Backman, Eds., 85921D-85921D-8 (2013).

[17] Brulle, Y., Bouchy, A., Valance, B., and André, J.C., "Industrial photochemistry XXI. Chemical, transport and refractive index effects in space-resolved laser photopolymerization,” Journal of Photochemistry and Photobiology A: Chemistry 83(1), 29-37 (1994).

[18] Lin-Gibson, S., Bencherif, S., Cooper, J.A., Wetzel, S.J., Antonucci, J.M., Vogel, B.M., Horkay, F., and Washburn, N.R., "Synthesis and characterization of PEG dimethacrylates and their hydrogels.," Biomacromolecules 5(4), 1280-7.

[19] Chan, S.C.W., Bürki, A., Bonél, H.M., Benneker, L.M., and Gantenbein-Ritter, B., "Papain-induced in vitro disc degeneration model for the study of injectable nucleus pulposus therapy.," The spine journal : official journal of the North American Spine Society 13(3), 273-83 (2013).

[20] Van Staveren, H.J., Moes, C.J., van Marie, J., Prahl, S. a, and van Gemert, M.J., "Light scattering in Intralipid$10 \%$ in the wavelength range of 400-1100 nm.,” Applied optics 30(31), 4507-14 (1991). 